Int. J. Dev. Biol. 62: 285-291 (2018)

https://doi.org/10.1387/ijdb.170167ds

\title{
Mutation of frizzled8a delays neural retinal cell differentiation and results in microphthalmia in zebrafish
}

\author{
XIAO-NING CHENG ${ }^{1}$, MING SHAO ${ }^{1}$ and DE-LI SHI*,2 \\ ${ }^{1}$ School of Life Sciences, Shandong University, Jinan, China and \\ ${ }^{2}$ Sorbonne Universités, UPMC Univ Paris 06, CNRS UMR7622, IBPS-Developmental Biology Laboratory, France
}

\begin{abstract}
Eye formation in vertebrates involves highly coordinated processes, and the differentiation of various eye tissues is regulated by conserved transcription factors and signalling pathways. Mutations in key genes of the regulatory hierarchy lead to congenital disorders and ocular diseases. The Wnt signalling pathway plays a key role in different aspects of eye development, and several Wnt receptors of the Frizzled family are required for eye specification and differentiation. However, their precise function in these processes remains elusive. Here we show that mutation of the frizzled8a gene in zebrafish leads to microphthalmia. The differentiation of retinal layers is delayed, and retinal progenitor cells in microphthalmic embryos fail to normally exit the cell cycle to enter into the post-mitotic state. They exhibit delayed differentiation associated with enhanced apoptosis, which results in abnormal lamination of retinal layers, reduction in the number of retinal cells, and small eye phenotype. These findings suggest that Frizzled8a plays a specific role in regulating cell cycle progression during the differentiation of retinal progenitor cells.
\end{abstract}

KEY WORDS: frizzled, retina, microphthalmia, cell cycle, zebrafish

\section{Introduction}

The vertebrate eye is a complex organ composed of three principle tissues: cornea, lens and retina. Wnt signalling regulates different aspects eye development (Graw, 2010). There are many lines of evidence indicating that the $\mathrm{Wnt} / \beta$-catenin pathway controls proliferation of retinal progenitor cells (RPCs) in various species (Kubo and Nakagawa, 2008; Denayer et al., 2008; Agathocleous et al., 2009; Borday et al., 2012), and is required for the maintenance of RPCs in a proliferative state (Meyers et al., 2012). Several Wnt receptors of the Frizzled $(\mathrm{Fz})$ family are shown to be implicated in these processes. In Xenopus, both $f z 3$ and $f z 5$ are expressed in the early optic vesicle, knockdown of $f z 3$ prevents the early induction of the eye field, while knockdown of $f z 5$ impairs cell proliferation in the developing retina (Shi et al., 1998; Sumanas and Ekker, 2001; Rasmussen et al., 2001; Van Raay et al., 2005). There is also evidence indicating that different $\mathrm{Fz}$ proteins may play both redundant and distinct function during eye development. In zebrafish, functional analyses suggest that $f z 8 a$ interacts with $w n t 8 b$ to antagonize eye specification, whereas $f z 5$ interacts with wnt11 to promote eye development (Kim et al., 2002; Cavodeassi et al., 2005). Nevertheless, whether they regulate the differentiation process has not been studied.
In this study, we examined the implication of $f z 8 a$ gene in retinal differentiation in zebrafish. Two $f z 8$ genes ( $f z 8 a$ and $f z 8 b$ ) are present in the zebrafish genome, but only $f z 8 a$ is expressed in the developing retina. We find that mutation of $f z 8 a$ leads to microphthalmia. The affected embryos display smaller eye size with impaired retinal differentiation. Noticeably, RPCs remain in a proliferative state, but they fail to exit the cell cycle and to undergo differentiation. As a consequence, retinal differentiation is delayed and the lamination of retinal layers is disorganized. These results suggest that Fz8a may be involved in regulating the cell cycle exit in RPCs during retinal differentiation.

\section{Results}

Loss-of-function of $\mathrm{fz} 8 \mathrm{a}$ in zebrafish reduces eye size

We used transcription activator-like effector nucleases (TALENs) genome-editing approach to inactivate fz8a gene and generated a

\footnotetext{
Abbreviations used in this paper: GMZ, ciliary marginal zone; Fz, Frizzled; Fz8a, Frizzled8a; GCL, ganglion cell layer; hpf, hours post-fertilisation; INL, inner nuclear layer; MZ, maternal-zygotic; ONL, outer nuclear layer; RPCs, retinal progenitor cells; RPE, retinal pigment epithelium; TALENs, transcription activatorlike effector nucleases.
}

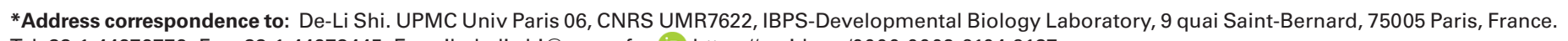
Tel: 33-1-44272772. Fax: 33-1-44273445. E-mail: de-li.shi@upmc.fr - (iD) https://orcid.org/0000-0002-6104-9137

Supplementary Material (four figures) for this paper is available at: http://dx.doi.org/10.1387/ijdb.170167ds

Submitted: 18 July, 2017; Accepted: 16 January, 2018.

ISSN: Online 1696-3547, Print 0214-6282 
A $f z 8 a$

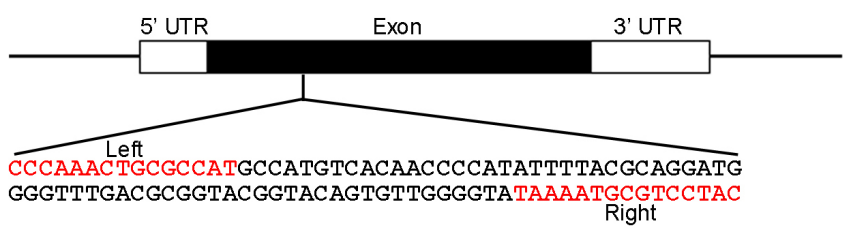

fz8a CGCCGATGGTGC. . . GCCATGTCACAACCCATA. . CGCCGGGCACGA

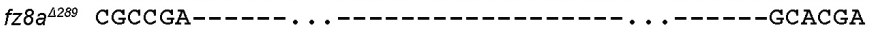

B

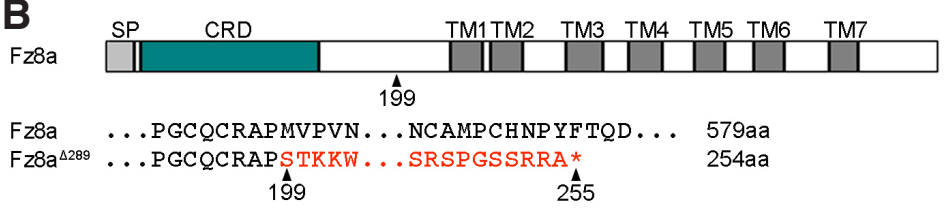

C
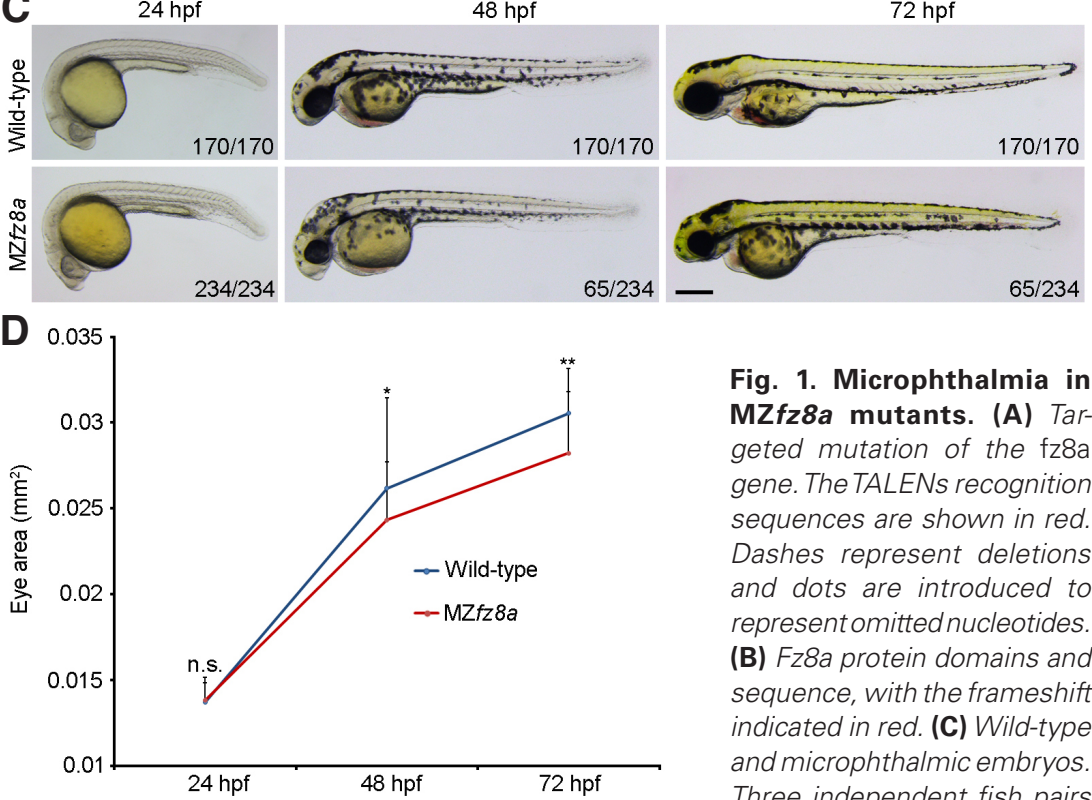

Fig. 1. Microphthalmia in MZfz8a mutants. (A) Targeted mutation of the fz8a gene. The TALENs recognition sequences are shown in red. Dashes represent deletions and dots are introduced to representomitted nucleotides. (B) Fzoa protein domains and sequence, with the frameshift indicated in red. (C) Wild-type and microphthalmic embryos. Three independent fish pairs were followed at indicated stages. (D) Statistics of eye size from two independent experiments ( ${ }^{*}, P<0.05 ;{ }^{* *}, P<0.01$; n.s., not significant). Scale bar, $300 \mu \mathrm{m}$. mutation with 289 nucleotides deletion in its unique exon (Fig. 1A). This leads to a frameshift after amino acid 198 and a premature stop codon after amino acid 254 (Fig. 1B). Genotyping indicated that both the gene and the corresponding transcript were truncated around the target sites (Supplementary Fig. S1).

Maternal-zygotic (MZ) fz8a (MZfz8a) mutants developed normally until the end of somite segmentation period (26 somites). At 24 hpf (hours post-fertilization), no morphological difference could be observed between wild-type and MZfz8a embryos. However, at $48 \mathrm{hpf}$, there was a clear reduction of the eye size in some MZfz8a mutants, and at $72 \mathrm{hpf}$, this became more evident (Fig. $1 C, D)$. Analysis from three independent fish pairs indicated that MZfz8a mutants exhibited smaller eye phenotype with $25 \%$ to $30 \%$ penetrance (Fig. 1C, Supplementary Fig. S2), and those embryos developed microphthalmia.

A detailed examination of microphthalmic embryos revealed eye development defects prior to the reduction of eye size. At $24 \mathrm{hpf}$, compared with wild-type embryos or normal siblings, they failed to close the choroid fissure (Fig. 2 A, A',A"). At 36 $\mathrm{hpf}$, the choroid fissure in microphthalmic embryos became closed, but the thickness of the retina was obviously reduced and there was an accumulation of blood around the lens (Fig. 2 B,B', B"), which resembles persistent fetal vasculature (PFV). During subsequent stages, it was evident that retina pigmentation and the eye size became reduced in these embryos (Fig. 2 C-E,C'-E',C'-E"). At 96 hpf, microphthalmic embryos also displayed lens development defects (Fig. 2F,F',F"). Since mutation of MZfz8a did not affect the specification of the eye field (Supplementary Fig. S3), these optic defects imply that $f z 8 a$ is required for eye differentiation.
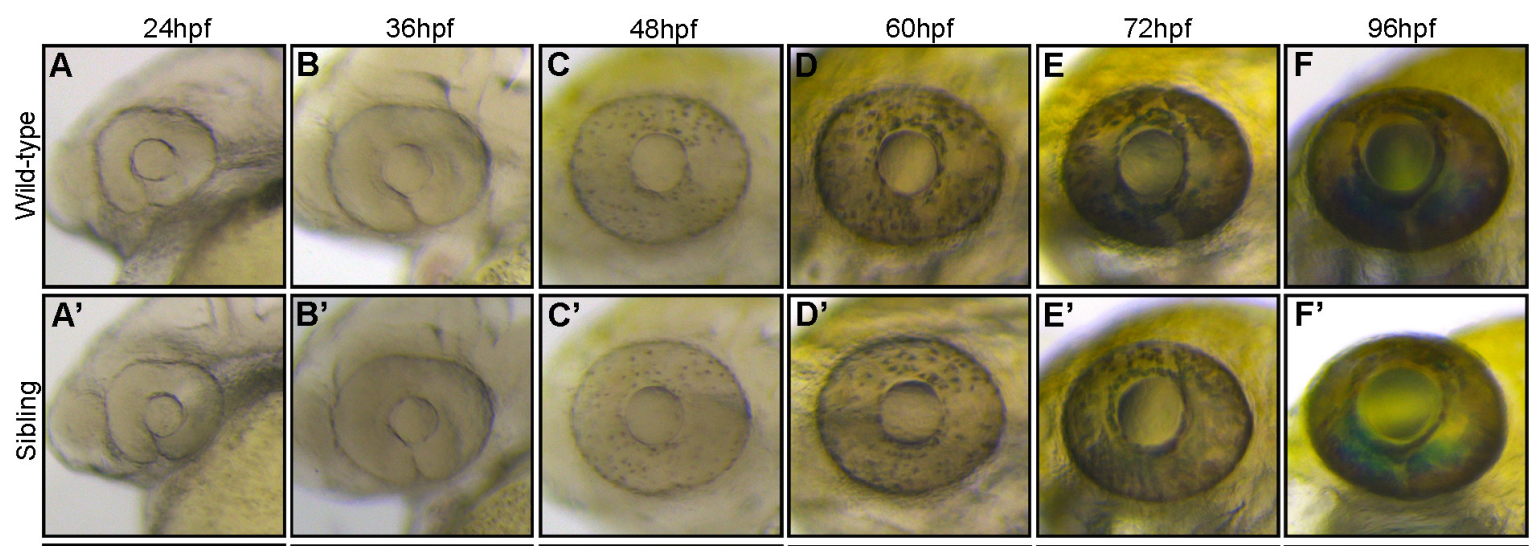

Fig. 2. Defective eye development in microphthalmic embryos. (A-F) Eye development in wild-type embryos. (A'F') MZfz8a siblings show normal eye development. (A"-F") Defective eye development in microphthalmic embryos. The retina is outlined at $24 \mathrm{hpf}$ to show the persistence of the choroid fissure (arrow). Arrowheads indicate blood
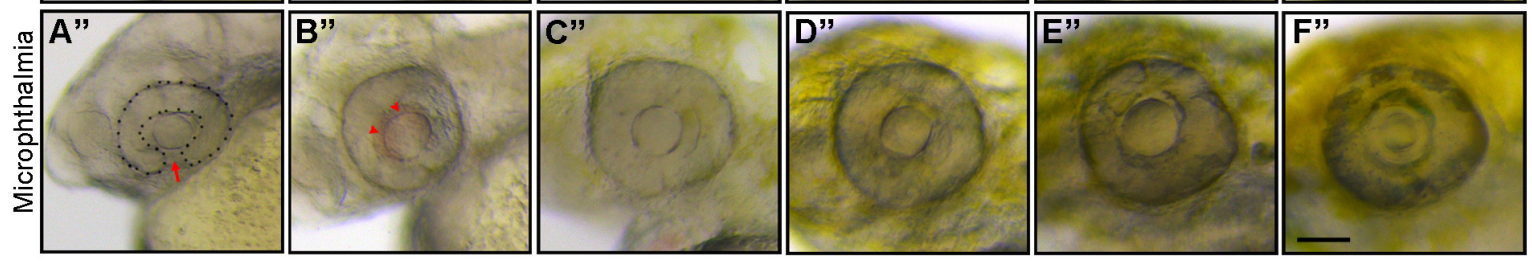
accumulation around the lens. A reduction of the retina and eye size is evident at 36 hpf, lens differentiation defect can be observed at $96 \mathrm{hpf}$. Scale bar, $100 \mu \mathrm{m}$. 

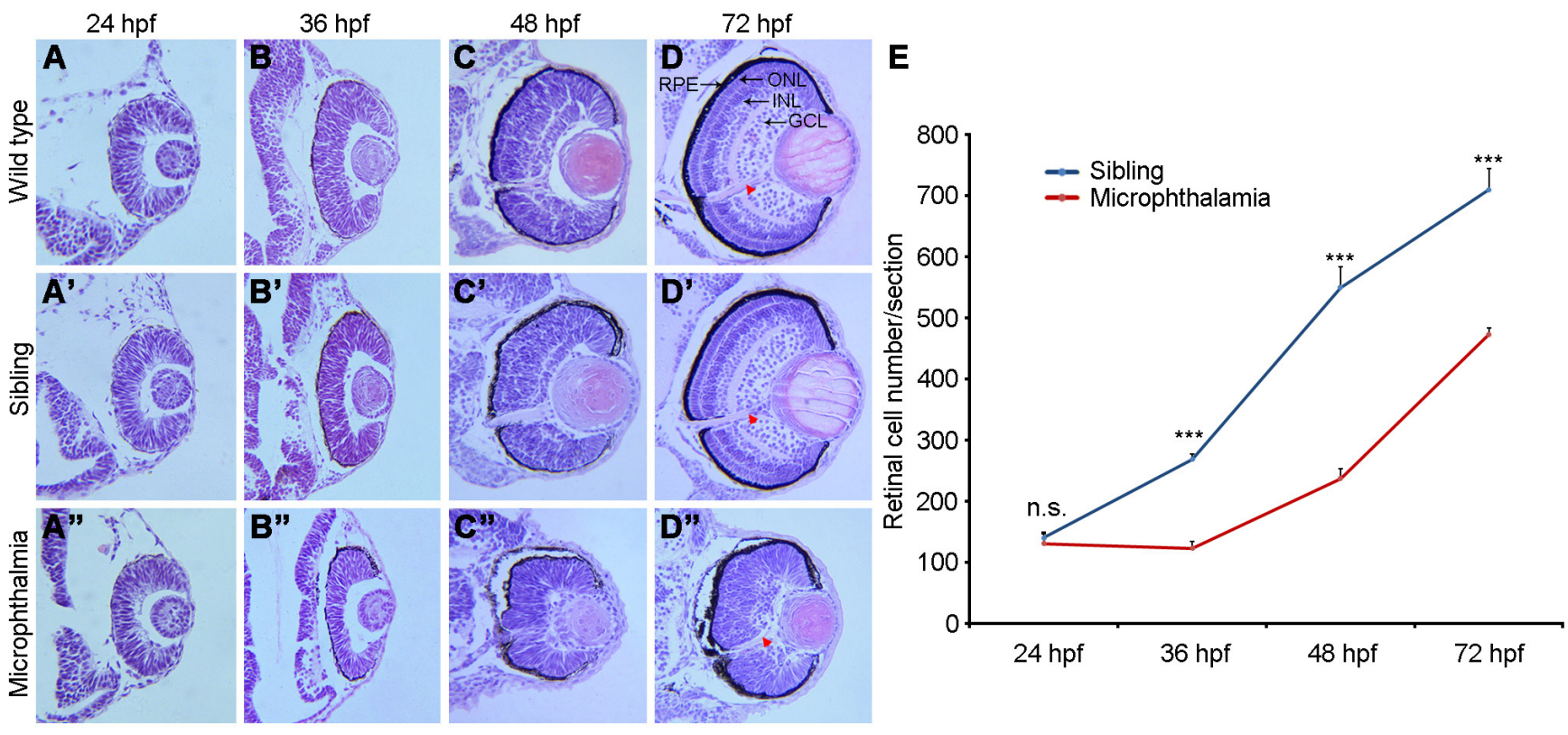

Fig. 3. Delayed lamination and reduced retinal cell number in microphthalmic mutants. (A-D) Retina structure and lamination in wild-type embryos. (A'-D') MZfz8a siblings show aspects of retina and lens differentiation which are similar to those of wild-type embryos. (A"-D") Microphthalmic embryos display disorganized retinal layers. The retina is thinner at $36 \mathrm{hpf}$ and pseudostratified at 48 hpf. GCL lamination can be distinguished at 72 hpf. Arrowheads indicate the optic nerve. (E) Statistics of retinal cells show the delay in cell number increase from $24 \mathrm{hpf}$ to $36 \mathrm{hpf}$. Bars represent the mean \pm s.d. from three sections (***, P<0.001; n.s., not significant). Scale bar: $50 \mu \mathrm{m}$.

\section{Microphthalmic embryos display delayed retinal lamination and reduced retinal cell numbers}

To examine how the eye size is reduced in microphthalmic embryos, we first performed histological analysis of eye structure. At $24 \mathrm{hpf}$, compared with wild-type embryos and normal siblings, no discernible alteration was observed in the microphthalmic retina (Fig. 3 A,A',A"). However, at $36 \mathrm{hpf}$, neural retina became obviously thinner and lens size was reduced, whereas the retinal pigment epithelium (RPE) seemed to be normally laminated (Fig. $\left.3 \mathrm{~B}, \mathrm{~B}^{\prime}, \mathrm{B}^{\prime \prime}\right)$. At $48 \mathrm{hpf}$, when the ganglion cell layer (GCL) became laminated in wild-type embryos and normal siblings, there was a clear disorganization of the neural retina in microphthalmic mutants, which was formed by a pseudostratified epithelium. The differentiation of lens fibers was also delayed, as revealed by the presence of nuclei (Fig. 3 C,C',C"). At $72 \mathrm{hpf}$, the outer nuclear layer $(\mathrm{ONL})$, inner cell layer (INL), and $\mathrm{GCL}$ in the neural retina were

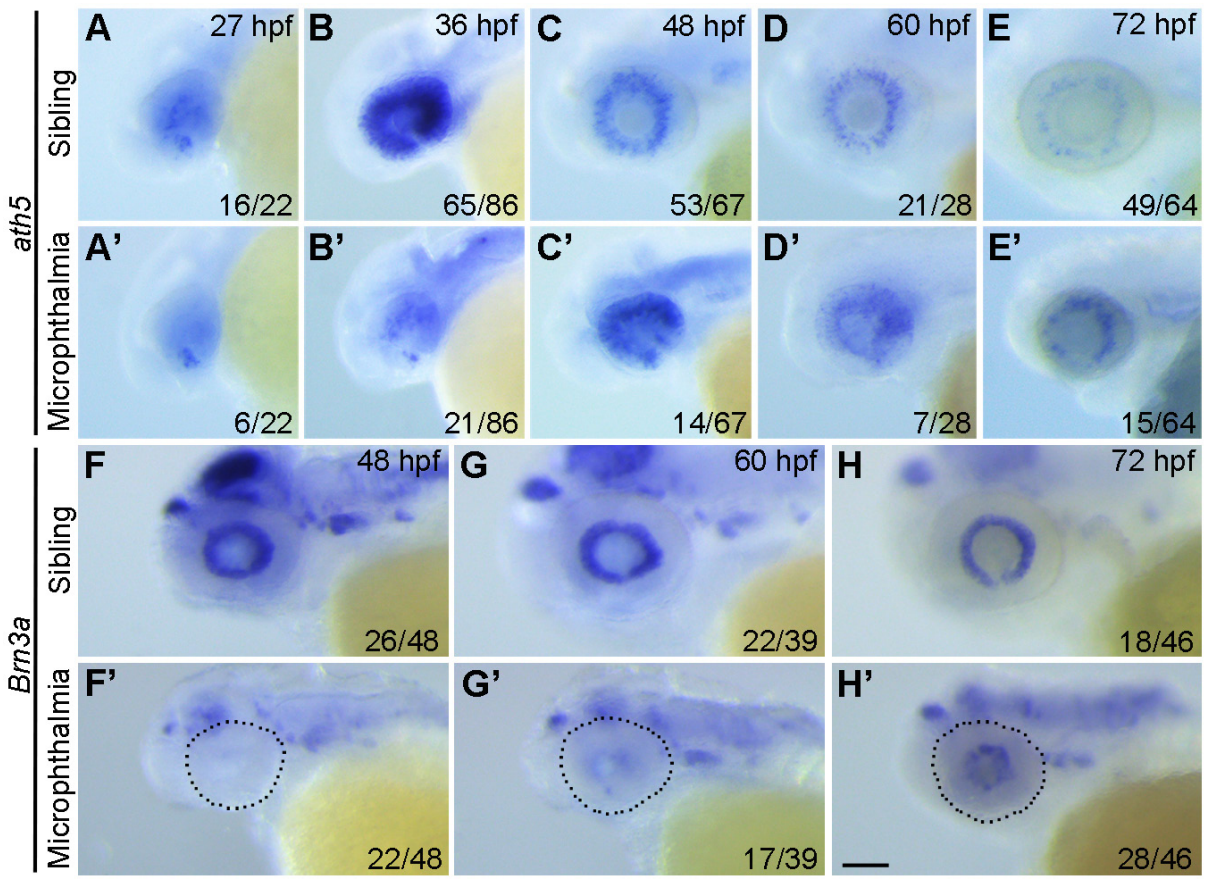
well differentiated and organized in wild-type and sibling eyes (Fig. 3 D, D'). However, the ONL and INL were not distinguishable, only some GCL cells began to be laminated and the optic nerve began to be differentiated in microphthalmic embryos (Fig. 3D"), indicating an impaired retinal lamination.

This apparent delay in retinal differentiation may be due to a reduced proliferation of neural retina cells. We thus compared the cell number in the neural retina between microphthalmic and normal sibling eyes. By examining comparable histological sections, we found that, in normal siblings, there was

Fig. 4. Neuronal differentiation is delayed in the microphthalmic retina. (A-E) The expression of ath5 in normal siblings picks at 36 hpfand becomes restricted to the retina ganglion progenitor cells from 48 hpfonward. (A'-E') The expression of ath5 in microphthalmic retina ganglion progenitor cells is shifted to $72 \mathrm{hpf}$. (F-H') Analysis of the expression of brn3a in the retina ganglion progenitor cells in normal sibling and microphthalmic eyes (outlined) shows a similar shift. Scale bar, $100 \mu \mathrm{m}$. 
a steady increase in retinal cell number from $24 \mathrm{hpf}$ to $72 \mathrm{hpf}$, while in microphthalmic embryos, there was no such increase between $24 \mathrm{hpf}$ and $36 \mathrm{hpf}$. During subsequent stages, although there was a similar kinetics of increase in retinal cell number between normal siblings and microphthalmic embryos, the total number of microphthalmic retinal cells remained significantly low (Fig. 3E). Although the three major neural retina cell layers eventually laminated at $96 \mathrm{hpf}$ to $120 \mathrm{hpf}$, they were significantly thicker compared to those in wild-type embryos or normal siblings (Supplementary Fig. S4). This indicates that mutation of $\mathrm{fz} 8 \mathrm{a}$ delays retinal cell differentiation.
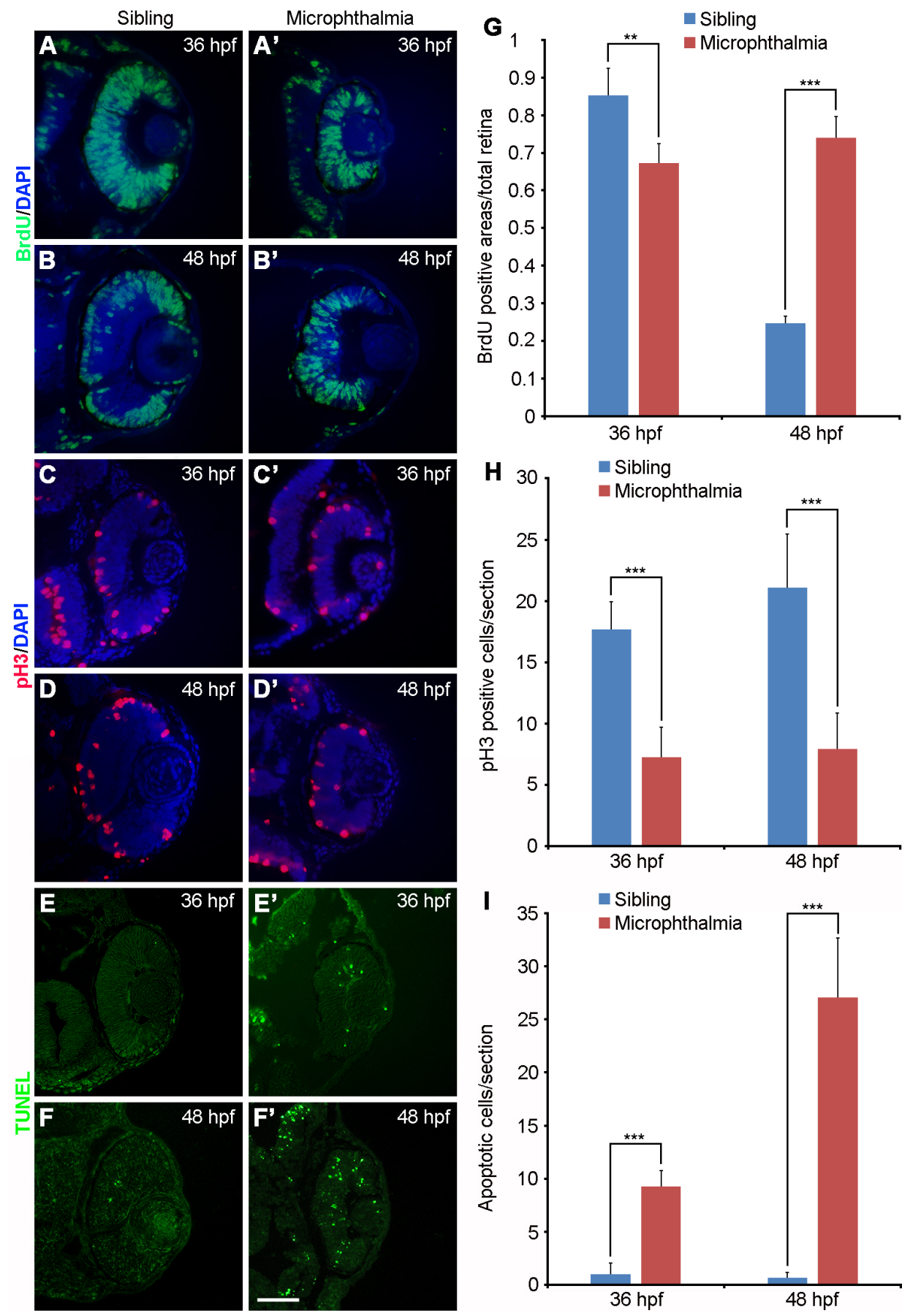

Both ath5 and brn3a are markers of neuronal production in the retina. We thus analyzed their expression to see how neuronal differentiation is affected. In normal siblings, ath5 expression was low at $27 \mathrm{hpf}$ but peaked at $36 \mathrm{hpf}$ (Fig. 4 A,B). It then decreased progressively to become restricted in the retinal ganglion progenitor cells (Fig. 4 C-E). In microphthalmic embryos, however, this temporal expression pattern exhibited a shift by at least 12 hours (Fig. 4 A'-E'). Interestingly, brn3a expression showed a similar decrease as ath5 in normal siblings from $48 \mathrm{hpf}$ to $72 \mathrm{hpf}$ (Fig. $4 \mathrm{~F}-\mathrm{H})$, while it began to be expressed at $60 \mathrm{hpf}$ and increased at 72 hpf in microphthalmic embryos (Fig. 4 F'-H'). These analyses clearly show that the differentiation of neural retina cells is delayed, but not abolished, in microphthalmic embryos.

\section{Abnormal retinal cell proliferation and apoptosis in microphthalmic embryos}

We next employed different markers to characterize molecularly the delayed retinal cell differentiation in microphthalmic embryos. Neural retina cells were incubated in BrdU for 30 minutes and then cultured for 2 hours. Whereas cell proliferation (BrdU incorporation) decreased in sibling eyes between $36 \mathrm{hpf}$ and $48 \mathrm{hpf}$ as retinal progenitors exited cell cycle to differentiate (Fig. 5A,B,G), we observed a maintenance in the number of proliferative retinal progenitor in microphthalmic eyes (Fig. 5 A', B', G), indicating a sustained retinal cell proliferation. However, since the total number of neural retina cells in microphthalmic embryos was low (see Fig. 3), the sustained proliferation may not be accompanied by an active cell division. Indeed, phosphorylated histone $\mathrm{H} 3(\mathrm{pH} 3)$ staining showed that, both at 36 hpf and $48 \mathrm{hpf}$, there were significantly less dividing cells, essentially localized at the apical side of the microphthalmic retina (Fig. 5 C-D',H). This indicates a reduced division rate of neural retina cells. However, the possibility of an increased apoptosis of RPCs cannot be excluded. Analysis by TUNEL assay indicated that

Fig. 5. Sustained proliferation and abnormal apoptosis of microphthalmic retinal cells. (A-

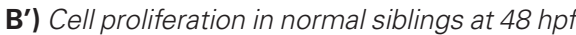
is restricted to the RPCs of the $C M Z$, while the entire microphthalmic retinal cells are proliferative. (C-D') Reduced pH3-positive dividing cells in microphthalmic embryos at $36 \mathrm{hpf}$ and $48 \mathrm{hpf}$. (E-F') Increased apoptotic microphthalmic cells at 36 hpf and 48 hpf. (G-I) Statistics of BrdUpositive area, $\mathrm{pH} 3$-positive cells, and apoptotic cells. All data were obtained from three sections at a comparable position between normal siblings and microphthalmic embryos ${ }^{*}{ }^{*}, P<0.01$; ***, $P<0.001$; n.s., not significant). Scale bar: $50 \mu \mathrm{m}$. 
very few fluorescent apoptotic spots were present in normal siblings (Fig. 5 E,F,I), by contrast, significantly increased apoptotic cells were observed in the microphthalmic embryos (Fig. 5 E',F',I). These observations suggest that reduced division rate and increased apoptosis in neural retina cells may account for the microphthamia phenotype.

\section{Cell cycle exit is delayed in microphthal- mic embryos}

The sustained proliferation state of RPCs in microphthalmic embryos is not consistent with the reduced division of these cells. One possibility that accounts for this discrepancy may be a delay of cell cycle exit. The expression of myca is correlated with retinal cell proliferation. We found that mycaexpression in the wild-type retina began to be predominantly localized to the ciliary marginal zone (CMZ) at $24 \mathrm{hpf}$, and became entirely restricted in the CMZ from $36 \mathrm{hpf}$ onward (Fig. 6 A-D). By contrast, it was detected nearly in the entire microphthalmic retina at $24 \mathrm{hpf}$ and $36 \mathrm{hpf}$ (Fig. 6 A',B'). The expression level of myca in the proximal region of microphthalmic retina began to decrease at $48 \mathrm{hpf}$, and restricted myca expression could be observed in the $\mathrm{CMZ}$ at $60 \mathrm{hpf}$ (Fig. 6 C',D'). Thus, both the pattern and level of myca expression in microphthalmic embryos show a delay of neural retina cell differentiation by about 24 hours, likely caused by a sustained proliferation of these cells.

Both cyclinD1 and the cyclin-dependent kinase inhibitor $\mathrm{p} 57^{\mathrm{Kip} 2}$ are important regulators of RPC proliferation. The expression of cyclinD1 becomes rapidly down-regulated in emerging post-mitotic cells, while conversely, the expression of p57Kip2 is up-regulated in a subset of RPCs as they exit the cell cycle (Das et al., 2009; Dyer and Cepko, 2001; Shkumatava and Neumann, 2005). At $24 \mathrm{hpf}$ and $36 \mathrm{hpf}$, similar intensity and distribution of cyclinD1 transcripts were detected in wild-type and microphthalmic embryos (Fig. 6E,F,E',F'). Interestingly, from $48 \mathrm{hpf}$ to $60 \mathrm{hpf}$, low level of cyclinD1 expression was detected in the $\mathrm{CMZ}$ of wild-type embryos (Fig. $6 \mathrm{G}, \mathrm{H}$ ). By contrast, high level of cyclinD1 expression was maintained in the entire microphthalmic retina (Fig. 6 G',H'). Complementary to cyclinD1, p57Kip2 was expressed at low level in the wild-type retina at $24 \mathrm{hpf}$ (Fig. 6l). After an increase in the proximal neural retina at 36 hpf (Fig. 6J), indicating that these cells exit the cell cycle, its expression decreased and became restricted in the RPCs of the $\mathrm{CMZ}$ at $48 \mathrm{hpf}$ and $60 \mathrm{hpf}$ (Fig. $6 \mathrm{~K}, \mathrm{~L}$ ). By contrast, p57Kip2 expression was not detected in the microphthalmic retina at $24 \mathrm{hpf}$ and $36 \mathrm{hpf}$
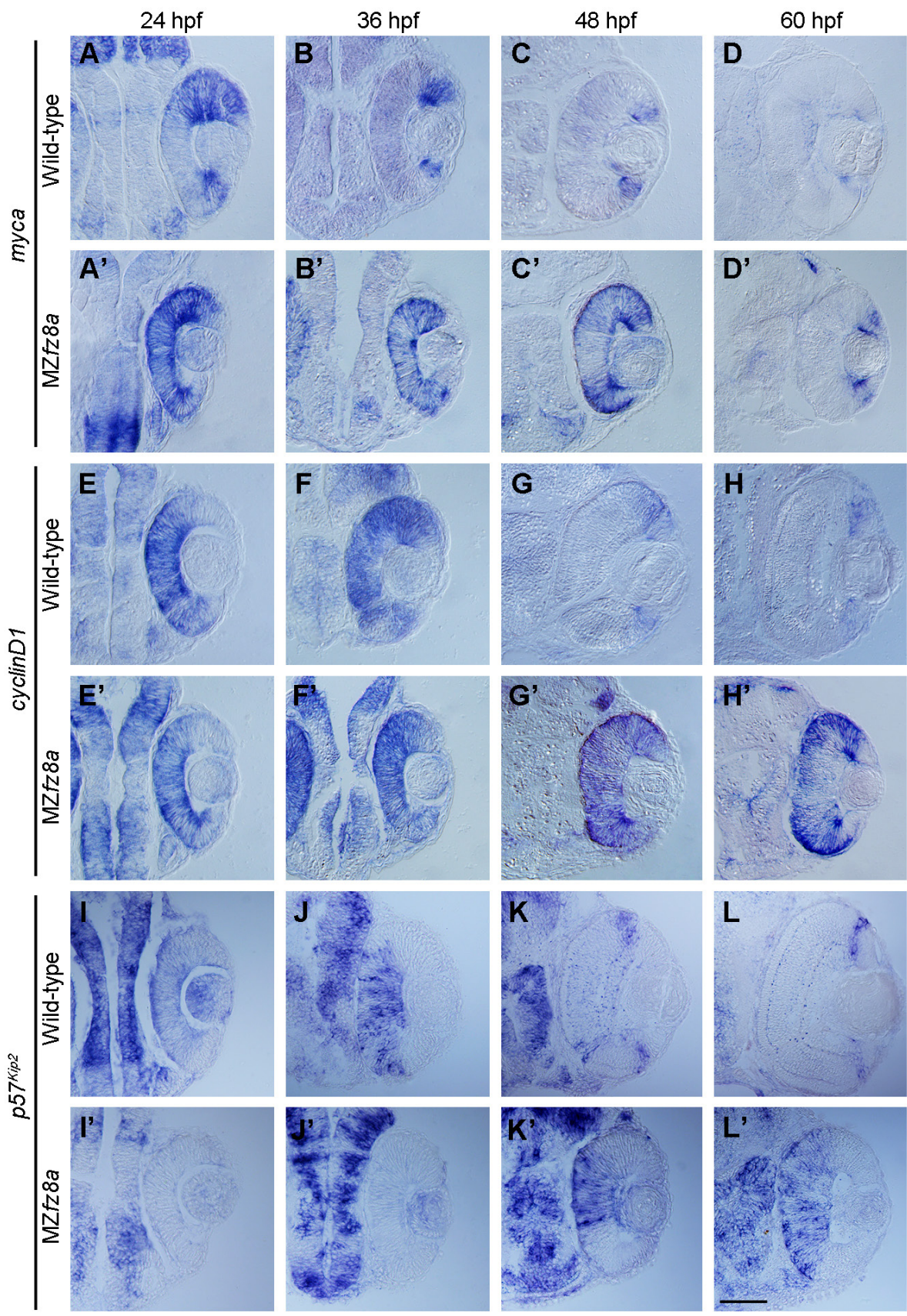

Fig. 6. Delayed cell cycle exit in microphthalmic embryos. (A-D') The expression of myca in wild-type embryos is restricted to RPCs of the CMZ from 36 hpf onward, while it persists in the entire microphthalmic retina at $36 \mathrm{hpf}$ and $48 \mathrm{hpf}$. (E-H) The expression of cyclinD1 in wild-type embryos is restricted to the RPCs of the CMZ at 48 hpf and 60 hpf. (E'-H') In microphthalmic embryos, cyclinD1 remains to be expressed in the entire retina at all stages examined. (I-L) The expression of p57kip2 in the wild-type retina increases at $36 \mathrm{hpf}$, and is progressively restricted in the RPCs of the CMZ from 48 hpf to $60 \mathrm{hpf}$. (I'-L') In microphthalmic embryos, the expression of p57 Kip2 increases at $48 \mathrm{hpf}$ and persists at $60 \mathrm{hpf}$. Scale bar: $50 \mathrm{\mu m}$. 
the cell cycle. As a result, neural differentiation is defective due to increased apoptosis and reduced amount of retinal cells.

\section{Discussion}

We show that knockout of $f z 8$ a results in microphthalmia in zebrafish. RPCs in these mutants have prolonged period of proliferation without increase in cell number. These cells exhibit a delayed exit from the cell cycle, which likely prevents them to enter into the post-mitotic state for neuronal differentiation.

In MZfz8a mutants, about $30 \%$ of the embryos progressively develop microphthalmia. This low penetrance could not due to the compensation by $f z 8 b$, which is not expressed in the developing eye. Rather, at least $f z 3$ and $f z 5$ are expressed early in the eye region (Shi et al., 1998; Cavodeassi et al., 2005). They may functionally interact with $f z 8 a$ to regulate eye development. Single knockout of $f z 5$ in mice results in mild coloboma and microphthalmia with about $50 \%$ penetrance (Liu and Nathans, 2008), while single knockout of $f z 8$ causes similar but weaker phenotypes (Liu et al., 2012). However, compound $f z 5$ and $f z 8$ mutant exhibits severe retinal coloboma and microphthalmia (Liu et al., 2012). Thus, the ocular defects caused by $f z 8$ loss-of-function are closely similar between zebrafish and mice, indicating a conserved function of $f z 8$ in eye development. There should be also a functional redundancy between Fz proteins that have similar ligand-binding and signalling specificity.

The Wnt pathway during eye development shows a high degree of complexity, depending largely on the cellular context. Our present work suggests that $f z 8 a$ is implicated in RPC proliferation and differentiation through regulation of cell cycle exit. Indeed, Wnt signalling regulates cell proliferation and cell cycle progression by activating target genes $c-m y c$ and $c y c l i n D 1$. Consistently, we find an altered expression of cyclinD1 in the microphthalmic RPCs. In particular, cyclinD1 expression is initially down-regulated in the RPCs, and is recovered with a significant delay. This indicates that RPCs remains in a mitotic state for a longer period, and as a consequence, their differentiation becomes delayed. Thus, we conclude that $f z 8 a$ functions in the step of neuronal production and differentiation.

The microphthalmic retina remains pseudostratified, with a significant delay of retinal lamination. This may be caused by a sustained proliferation of neural retina cells and/or by a delayed cell cycle exit. As a result, these cells are maintained in an undifferentiated state for a prolonged period. However, the process that shifts neuronal differentiation to later stages of eye development may be more complex. Indeed, the increased proliferation of retinal cells and delayed cell cycle exit are also accompanied by an enhanced apoptosis. Furthermore, although pH3 staining is essentially localized to the apical surface of the microphthalmic retina, it clearly shows a reduced number of dividing cells. Thus, the decrease of cell division associated with an increase of apoptotic cells may account for the reduction of retinal cells and for the microphthalmia phenotype. Nevertheless, it remains to be determined whether those non-dividing cells eventually enter into apoptosis.

The phenotype of microphthalmic MZfz8a embryos also resembles PFV. Since this occurs earlier than the appearance of abnormal retinal cell proliferation and apoptosis, it suggests that Fz8a may regulate other aspects of eye development. Thus, further analysis of its function may help to understand the cause of human ocular disease.

\section{Materials and Methods}

\section{Zebrafish embryos}

Zebrafish embryos were cultured at $28.5^{\circ} \mathrm{C}$. Phenylthiourea (SigmaAldrich) at $50 \mu \mathrm{M}$ was added to E3 solution to prevent pigment development.

\section{Targeted mutation}

TALEN recognition sequences are located in the 5 ' region of the unique exon of $f z 8 a$ locus (see Fig. 1). The targeting efficiency was determined as described (Cheng et al., 2017). Genotyping PCR primers are as follow:

F1, 5'-ATGAGGTGCGATCTACTGC-3'

F2, 5'-ACCAACTACCCCAGCAAAG-3'

Fw, 5'-CGCTCTACAACCGCGTTAAG-3'

R3, 5'-GAAGTAGATAAGCAGGAACAC-3'

R4, 5'-TGCTCCACGTCATACTCCC-3' and

R5, 5'-GTTGCCAACGTAGCAGATC-3'.

\section{In situ hybridization and RT-PCR}

In situ hybridization was performed as described (Shao et al., 2017). Antisense probes were labelled using digoxigenin-11-UTP. Wild-type and mutant $f z 8 a$ transcripts were analyzed by semi-quantitative PCR using primers $\mathrm{F} 2$ and $\mathrm{R} 4$, with $\beta$-actin ( $5^{\prime}$-CACAGTGCTGTCTGGAGGTAC-3', 5'-GAGGGCAAAGTGGTAAACG-3') as a loading control.

\section{Histology and immunofluorescence}

Embryos were fixed in 4\% paraformaldehyde for 2 hours at room temperature, and were embedded in paraffine. Sections of $5 \mu \mathrm{m}$ were made and stained with hematoxylin and eosin (HE). For immunofluorescence, sections were incubated with G3G4 monoclonal anti-BrdU antibody (Developmental Studies Hybridoma Bank, 1:300), or anti-pH3 antibody (Abcam, 1:1000), followed by Alexa Fluor488 rabbit anti-mouse IgG or Fluo594 goat anti-rabbit IgG (Interchim, 1:1000). They were analyzed under a fluorescence microscope (Leica DM2000).

\section{BrdU incorporation, cell proliferation and apoptosis assays}

Embryos were dechorionated and incubated in Ringer's solution containing $10 \mathrm{mM} \mathrm{BrdU} \mathrm{(Roche} \mathrm{Diagnostics)} \mathrm{and} \mathrm{15 \%} \mathrm{dimethylsulfoxide}$ for 30 minutes at $6^{\circ} \mathrm{C}$. They were washed 3 times with Ringer's solution (10 minutes each) and recovered at $28.5^{\circ} \mathrm{C}$ for at least 2 hours, and were then fixed with $4 \%$ paraformaldehyde overnight at $4^{\circ} \mathrm{C}$ for paraffine sectioning. Detection of mitotic cells was performed through anti-pH3 antibody staining. Labelling for apoptosis was performed using the TUNEL kit (Roche Diagnostics).

For each of the staining described above, at least 3 retinal sections at a comparable position in wild-type and mutant embryos from different experiments were selected. BrdU-positive area relative the entire neural retina surface, $\mathrm{pH} 3$-positive cells, and TUNEL-labelled fluorescent apoptotic bodies were analyzed.

\section{Retinal cell number counting}

At each stage, 3 retinal sections from sibling and mutant embryos at similar position of the eye were identified by the presence of optic nerve.

\section{Determination of eye size}

Synchronously spawned wild-type and mutant embryos (50 at each stage) were fixed and individually imaged at the eye region, along with a known reference area. The eye size was analyzed by ImageJ (NIH Image) and the real size was calculated by comparison with the reference area.

\section{Determination of neural retina layer width}

The total width of the retina and the width of each retinal layer were measured in sectioned embryos at $120 \mathrm{hpf}$ from three independent spawning using a total of 20 sections with optic nerve. 


\section{Acknowledgements}

We thank members of our laboratory for technical assistance and zebrafish care. This research was supported by the NSFC grant (31671509).

\section{References}

AGATHOCLEOUS, M., IORDANOVA, I., WILLARDSEN, M.I., XUE, X.Y., VETTER, M.L., HARRIS, W.A. and MOORE, K.B. (2009). A directional Wnt/beta-cateninSox2-proneural pathway regulates the transition from proliferation to differentiation in the Xenopus retina. Development 136: 3289-3299.

BORDAY, C., CABOCHETTE, P., PARAIN, K., MAZURIER, N., JANSSENS, S., TRAN, H.T., SEKKALI, B., BRONCHAIN, O., VLEMINCKX, K., LOCKER, M. and PERRON, M. (2012). Antagonistic cross-regulation between Wnt and Hedgehog signalling pathways controls post-embryonic retinal proliferation. Development 139: 3499-3509.

CHENG, X.N., SHAO, M., LI, J.T., WANG, Y.F., QI, J., XU, Z.G. and SHI, D.L. (2017). Leucine repeat adaptor protein 1 interacts with Dishevelled to regulate gastrulation cell movements in zebrafish. Nat Commun 8: 1353.

CAVODEASSI, F., CARREIRA-BARBOSA, F., YOUNG, R.M., CONCHA, M.L., ALLENDE, M.L., HOUART, C., TADA, M. and WILSON, S.W. (2005). Early stages of zebrafish eye formation require the coordinated activity of Wnt11, Fz5, and the Wnt/beta-catenin pathway. Neuron 47: 43-56.

DAS, G., CHOI, Y., SICINSKI, P. and LEVINE, E.M. (2009). Cyclin D1 fine-tunes the neurogenic output of embryonic retinal progenitor cells. Neural Dev 4: 15.

DENAYER, T., LOCKER, M., BORDAY, C., DEROO, T., JANSSENS, S., HECHT, A., VAN ROY, F., PERRON, M. and VLEMINCKX, K. (2008). Canonical Wnt signaling controls proliferation of retinal stem/progenitor cells in postembryonic Xenopus eyes. Stem Cells 26: 2063-2074.

DYER, M.A. and CEPKO, C.L. (2001). p27Kip1 and p57Kip2 regulate proliferation in distinct retinal progenitor cell populations. J Neurosci 21: 4259-4271.

GRAW, J. (2010). Eye development. Curr Top Dev Biol 90: 343-386.
KIM, S.H., SHIN, J., PARK, H.C., YEO, S.Y., HONG, S.K., HAN, S., RHEE, M., KIM C.H., CHITNIS, A.B. and HUH, T.L. (2002). Specification of an anterior neuroectoderm patterning by Frizzled8a-mediated Wnt8b signalling during late gastrulation in zebrafish. Development 129: 4443-4455.

KUBO, F. and NAKAGAWA, S. (2008). Wnt signaling in retinal stem cells and regeneration. Dev Growth Differ 50: 245-251.

LIU, C. and NATHANS, J. (2008). An essential role for frizzled 5 in mammalian ocular development. Development 135: 3567-3576.

LIU, C., BAKERI, H., LI, T. and SWAROOP, A. (2012). Regulation of retinal progenitor expansion by Frizzled receptors: implications for microphthalmia and retinal coloboma. Hum Mol Genet 21: 1848-1860.

MEYERS, J.R., HU, L., MOSES, A., KABOLI, K., PAPANDREA, A. and RAYMOND, P.A. (2012). $\beta$-catenin/Wnt signaling controls progenitor fate in the developing and regenerating zebrafish retina. Neural Dev 7: 30.

RASMUSSEN, J.T., DEARDORFF, M.A., TAN, C., RAO, M.S., KLEIN, P.S. and VETTER, M.L. (2001). Regulation of eye development by frizzled signaling in Xenopus. Proc Natl Acad Sci USA 98: 3861-386.

SHAO, M., WANG, M., LIU, Y.Y., GE, Y.W., ZHANG, Y.J. and SHI, D.L. (2017). Vegetally localised $V$ rtn functions as a novel repressor to modulate $b m p 2 b$ transcription during dorsoventral patterning in zebrafish. Development 144: 3361-3374.

SHI, D.L., GOISSET, C. and BOUCAUT, J.C. (1998). Expression of Xfz3, a Xenopus frizzled family member, is restricted to the early nervous system. Mech Dev 70: 35-47.

SHKUMATAVA, A. and NEUMANN, C.J. (2005). Shh directs cell-cycle exit by activating p57Kip2 in the zebrafish retina. EMBO Rep 6: 563-569.

SUMANAS, S. and EKKER, S.C. (2001). Xenopus frizzled-5: a frizzled family member expressed exclusively in the neural retina of the developing eye. Mech Dev 103: $133-136$

VANRAAY,T.J., MOORE, K.B., IORDANOVA, I., STEELE, M., JAMRICH, M., HARRIS, W.A. and VETTER, M.L. (2005). Frizzled 5 signaling governs the neural potential of progenitors in the developing Xenopus retina. Neuron 46: 23-36. 


\section{Further Related Reading, published previously in the Int. J. Dev. Biol.}

Expression of Frizzleds and secreted frizzled-related proteins (Sfrps) during mammalian lens development Yongjuan Chen, Richard J.W. Stump, Frank J. Lovicu and John W. McAvoy

Int. J. Dev. Biol. (2004) 48: 867-877

http://www.intjdevbiol.com/web/paper/041882yc

Regulation of convergent extension in Xenopus by Wnt5a and Frizzled-8 is independent of the canonical Wnt pathway J B Wallingford, K M Vogeli and R M Harland

Int. J. Dev. Biol. (2001) 45: 225-227

http://www.intjdevbiol.com/web/paper/11291850

Crescent, a novel chick gene encoding a Frizzled-like cysteine-rich domain, is expressed in anterior regions during early embryogenesis P L Pfeffer, E M De Robertis and J C Izpisua-Belmonte

Int. J. Dev. Biol. (1997) 41: 449-458

http://www.intjdevbiol.com/web/paper/9240561

Ras-Related Nuclear Protein is required for late developmental stages of retinal cells in zebrafish eyes

Cheng-Yung Lin, Hsing-Yen Huang, Po-Nien Lu, Chien-Wei Lin, Kuang-Ming Lu and HuaiJen Tsai

Int. J. Dev. Biol. (2015) 59: 435-442

https://doi.org/10.1387/ijdb.150310ht

Cloning and developmental expression of zebrafish pdzrn3

Luciana Dente, Gaia Gestri, Michael Tsang, Tetsuhiro Kudoh, Stephen W. Wilson, Igor B. Dawid and Massimiliano Andreazzoli

Int. J. Dev. Biol. (2011) 55: 989-993

https://doi.org/10.1387/ijdb.113437ld

A moving wave patterns the cone photoreceptor mosaic array in the zebrafish retina Pamela A. Raymond and Linda K. Barthel

Int. J. Dev. Biol. (2004) 48: 935-945

http://www.intjdevbiol.com/web/paper/041873pr

Genetics of photoreceptor development and function in zebrafish

Motokazu Tsujikawa and Jarema Malicki

Int. J. Dev. Biol. (2004) 48: 925-934

http://www.intjdevbiol.com/web/paper/041890mt

5 yr ISI Impact Factor $(2016)=2.421$
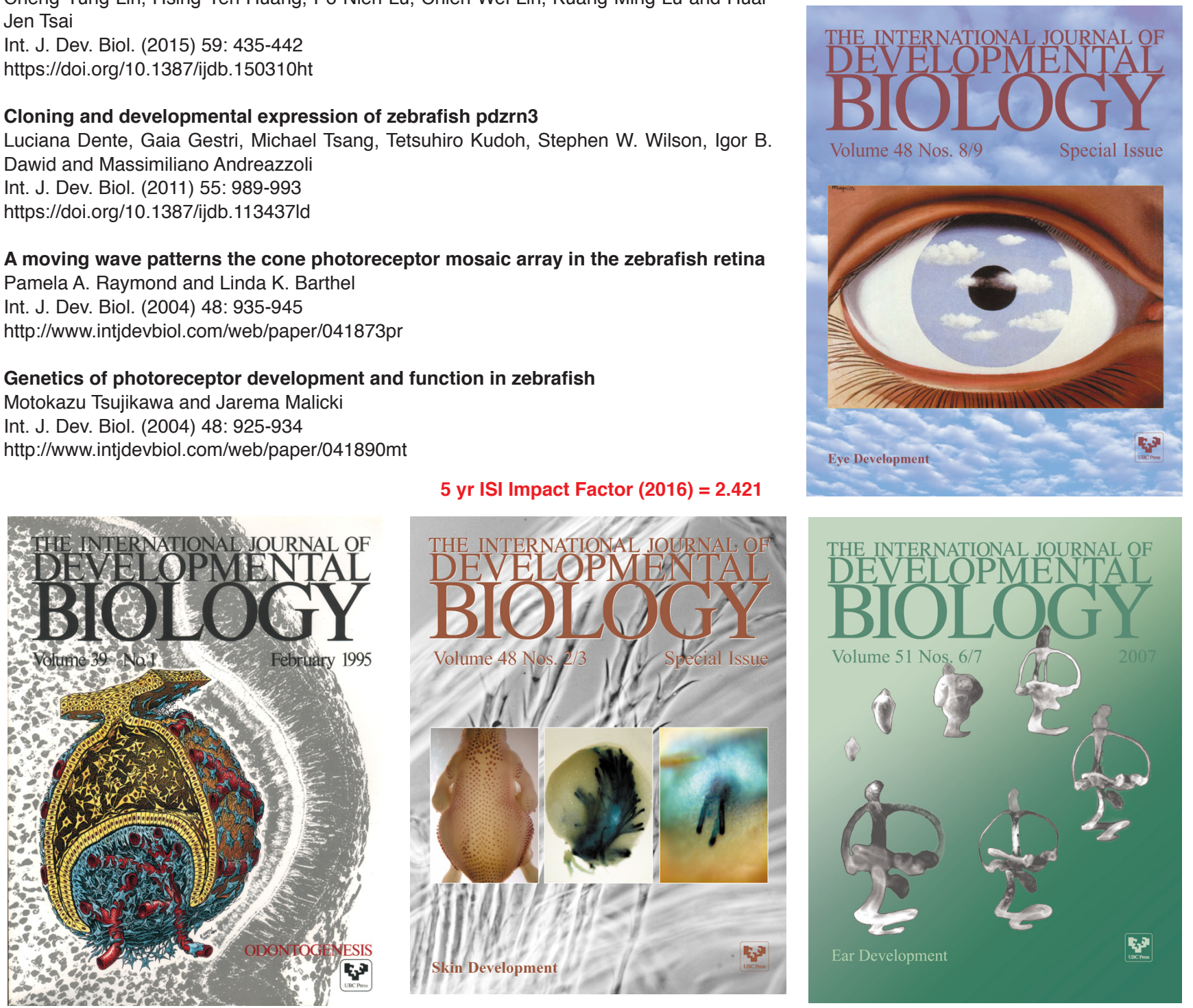